\title{
Prueba de tolerancia oral a la glucosa modificada en puérperas como diagnóstico retrospectivo de diabetes gestacional
}

\author{
Joel Cárdenas ${ }^{1}$, Leonor Arroyo ${ }^{1}$ \\ ${ }^{l}$ Instituto Especializado Materno Perinatal, Lima-Perú.
}

\begin{abstract}
Resumen
OBJETIVO: Determinar la relación entre la prueba de tolerancia oral a la glucosa (PTOG) en puérperas con macrosomia fetal y plantearla como método de diagnóstico retrospectivo para diabetes gestacional. DISEÑO DE ESTUDIO: Estudio clínico, de corte transversal, analítico, casos y controles. MATERIAL Y MÉTODOS: Estudio de 142 puérperas, 71 con recién nacidos macrosómicos y 71 con recién nacidos con pesos en percentiles de la normalidad. Se realizó una PTOG modificada de dos horas (basal, $1 \mathrm{~h}$ y $2 \mathrm{~h}$ ), con $100 \mathrm{~g}$ de glucosa, entre las primeras 8 y 48 horas posparto (positivo si [glicemia $2 \mathrm{~h}-$ basal] + [glicemia $1 \mathrm{~h}-$ basal] $>110$ $\mathrm{mg} / \mathrm{dL}$ ). Se utilizó el SPSS 10,0 para crear la base de datos y realizar el análisis estadístico: análisis univariado, bivariado para evaluar la hipótesis, y finalmente un análisis multivariado. RESULTADOS: La PTOG anormal resultó un factor de riesgo para macrosomia fetal, con un odds ratio ajustado de 5,9; IC95\% 2,6-13,4. Se encontró asociación entre antecedente de macrosomia fetal, odds ratio 15,7; IC95\% 1,9-123,6 y control prenatal odds ratio 5,7; IC95\% 1,2-26,8. CONCLUSIÓN: Existe relación entre la PTOG en puérperas y macrosomia fetal, que puede ser usada como prueba diagnóstica retrospectiva de diabetes gestacional.
\end{abstract}

Palabras clave: Test de tolerancia a la glucosa; macrosomia fetal; glucosa; diabetes gestacional; embarazo en diabetes.

Postpartum oral glucose tolerance test in mothers of macrosomic infants as a retrospective diagnostic method of gestational diabetes

Summary

OBJECTIVE: To assess the relationship between the postpartum oral glucose tolerance test (PPOGTT) and fetal macrosomia and its usefulness as a retrospective diagnostic method of gestational diabetes. DESIGN: Prospective case -control study. MATERIAL AND METHODS: Mothers with macrosomic infants $(\mathrm{n}=71)$ and controls $(n=71)$ were studied. PPOGTT was determined in all of them using 100 grams of glucose 8 to 48 hours postpartum. Data processing used SPSS 10,0. RESULTS: Abnormal PPOGTT is a risk factor to fetal macrosomia with an adjusted odds ratio (OR) 5,9 (95\% CI 2,6-13,4). There is association with a previous history of macrosomia OR 15,7 (95CI 1,9-123,6) and with prenatal control OR 5,7 (95\%CI 1,2-26,8).

CONCLUSIONS: PPOGTT may identify a subset of women with macrosomic infants who had gestational diabetes; we may consider it a retrospective diagnostic test.

Key words: Glucose tolerance test; fetal macrosomia; glucose; diabetes, gestational; pregnancy in diabetes.

Correspondencia:

Dr. Segundo Joel Cárdenas Goicoechea

Av. Faucett Cdra. 32, Mz K-19. Urb. Aeropuerto

Callao 1, Perú.

E-mail: joelcardenas@yahoo.com 


\section{INTRODUCCIÓN}

La diabetes gestacional complica 1,4 a $12 \%$ de todos los embarazos, dependiendo de los métodos de despistaje y diagnóstico utilizados en diferentes poblaciones $\left(^{(-5}\right)$. Es conocida la asociación entre diabetes gestacional y macrosomia fetal y se ha hallado una correlación con el desarrollo posterior de diabetes mellitus tipo II ${ }^{(6)}$.

Dado que la intolerancia a los carbohidratos durante el embarazo es asintomática en la mayoría de casos, solamente la búsqueda insistente del estado bioquímico con relación al metabolismo de los hidratos de carbono, nos llevará a mayores tasas de diagnóstico precoz de diabetes gestacional, así como, disminuir la morbilidad materno fetal, donde las consecuencias para el feto son más graves que las maternas. De ellas, la más importante es la macrosomia, debido a la dificultad de su tratamiento y a las controversias que existen con relación a su manejo $\left({ }^{7,8}\right)$. La morbilidad fetal constituye el parámetro más valioso del resultado del nacimiento de un feto macrosómico. Los riesgos son los relacionados con el proceso del parto que causan traumatismo neonatal, como la distocia de hombros, donde la parálisis del plexo braquial es casi inevitable y se presenta con una tasa de 2,2 por 1000 macrosómicos $\left(^{9}\right)$.

La diabetes gestacional incrementa también el riesgo neonatal de alteraciones metabólicas, prematurez, enfermedad de membrana hialina y aumenta la tasa de malformaciones congénitas $\left({ }^{10,11}\right)$. Los recién nacidos de madre diabética tienen un mayor riesgo de obesidad y de ser diabéticos en el futuro $\left({ }^{12}\right)$.

En el Instituto Especializado Materno Perinatal (IEMP) existiría un subregistro oficial de la incidencia de diabetes gestacional institucional. Pese a ser un centro de referencia nacional; no existe un consenso para su diagnóstico ni la búsqueda exhaustiva de esta patología, a pesar de la importancia ya descrita. Por ello es necesaria la identificación de estas pacientes en la etapa inmediatamente posterior al parto (puerperio), porque es conocido que las mujeres con antecedente de un recién nacido macrosómico tienen un riesgo tres veces mayor $\left({ }^{13-15}\right)$ de presentar otro(s) recién nacido macrosómico en los subsiguientes embarazos y con resultados perinatales adversos y alta morbilidad $\left(^{(, 9,11}\right)$. Además, las mujeres que desarrollan diabetes durante el embarazo tienen un riesgo alto (60$100 \%$ ) de desarrollar diabetes mellitus 12 a 18 años después $\left({ }^{16}\right)$.

No se ha hallado trabajos nacionales que asocien macrosomia y diabetes gestacional, pero sí investigaciones de cada uno de las variables señaladas en forma independiente, como los de Román $\left({ }^{13}\right)$, Pacora $\left({ }^{17,18}\right)$, Ardiles $\left({ }^{19}\right)$.

Planteamos como objetivo determinar la respuesta a la prueba de tolerancia oral a la glucosa modificada en puérperas con recién nacidos macrosómicos y proponer esta medición como una prueba indirecta para la cuantificación de la magnitud de la diabetes gestacional en nuestro medio.

\section{MATERIAL Y MÉTODOS}

Estudio de Investigación aplicada, cuyo diseño fue observacional, transversal, de casos y controles. El cálculo del tamaño muestral se realizó mediante el paquete estadístico EPI INFO versión 6, bajo los siguientes supuestos: Tipo de estudio: caso control no pareado. Nivel de confianza $=$ $95 \%$. Poder $=80 \%$. Proporción caso: control $=1$ $: 1$.

Muestra mínima a considerar para el trabajo fue 71 casos y 71 controles. Las variables dependientes fueron caso (puérperas con recién nacido macrosómico) y control (puérpera con un recién nacido de peso normal), y la variable independiente, prueba de tolerancia oral a la glucosa, aplicando la prueba de Carpenter.

Los criterios de inclusión fueron:

- Caso: Puérperas con recién nacido macrosómico $>4000$ gramos $\left({ }^{0}\right)$.

- Control: Puérpera con recién nacido a término 
con peso adecuado para la edad gestacional.

- Tiempo máximo desde el parto hasta el momento de la prueba: 48 horas

- Tiempo mínimo desde el parto hasta el momento de la prueba: 8 horas

- Hemodinámicamente estable

- Tolerar la ingesta de la solución glucosada.

Los criterios de exclusión fueron:

- Puérperas que tienen el antecedente de diabetes mellitus previo al embarazo.

- Pacientes con infusión de dextrosa $(5 \%, 33 \%)$.

- Pacientes que no tuvieran un ayuno mínimo de 8 horas.

- Cualquier foco infeccioso activo - Síndrome febril.

- Hemoglobina menor a $8 \mathrm{~g} \%$.

Identificada la paciente en los Servicios de Hospitalización, Centro Obstétrico y Centro Quirúrgico del IEMP y previo consentimiento informado, se extrajo la muestra de sangre venosa que constituiría la basal. Luego de ello, se administró $100 \mathrm{~g}$ de glucosa anhidra diluida en 200 $\mathrm{mL}$ de agua hervida y con el jugo de un limón mediano; transcurrida 1 hora se extrajo la segunda muestra y 2 horas después se extrajo la tercera muestra. El procesamiento de las muestras se realizó en el laboratorio de emergencia del IEMP. Se les aplicó la prueba validada por Carpenter para obtener el resultado final de glicemia: (glicemia 1hora - glicemia ayunas $)+($ glicemia $2 \mathrm{~h}-$ glicemia ayunas); se consideró positiva la prueba si se obtenía valores por encima o igual a $110 \mathrm{mg} / \%$ $\left({ }^{21}\right)$.

Se empleó el paquete SPSS para Windows versión 10,0. La información fue evaluada en un proceso escalonado de análisis, partiendo del análisis univariado, luego el análisis bivariado, donde se evaluó nuestra hipótesis. Se obtuvo valores crudos y ante la posibilidad de variables que produjeran interacciones o actuaran como confusores, se realizó el análisis multivariado, para determinar el peso de cada unos de los factores asociados con la macrosomia fetal.

\section{RESULTADOS}

Nuestra población de estudio consistió de 142 puérperas cuyo parto se produjo en el IEMP en el periodo de abril a junio de 2003. Los casos y controles fueron 71 para cada grupo. Las características generales de la población de estudio se muestra en la Tabla 1; Las características obstétricas se las observa en la Tabla 2.

En la Tabla 3 se presenta la validación de la hipótesis la prueba de tolerancia oral a la glucosa anormal (positivo) es mayor en los casos que en los controles, encontrándose positivo en 35 casos y 10 en los controles. Esta diferencia es estadísticamente significativa para un $\mathrm{p}=0,000$. Se obtuvo un OR crudo de 5,9 con un intervalo de confianza al $95 \%$ de 2,6-13,4.

Tabla 1. Características generales de la población de estudio.

\begin{tabular}{lcc}
\hline Característica & $\begin{array}{c}\text { Controles } \\
\text { nomacrosómicos }\end{array}$ & $\begin{array}{c}\text { Casos } \\
\text { macrosómicos }\end{array}$ \\
\hline Tamaño muestral (n) & 71 & 71 \\
Edad paciente & & \\
(años, promedio $\pm \mathrm{DE})$ & $25,9 \pm 5,8$ & $28,2 \pm 6,0$ \\
Edad de pareja & $29,3 \pm 7,2$ & $30,9 \pm 6,9$ \\
Peso inicial (kg) & $57,9 \pm 12,5$ & $64,0 \pm 11,4$ \\
Peso final (kg) & $68,2 \pm 11,6$ & $76,5 \pm 11,4$ \\
Variación del peso (kg) & $10,3 \pm 4,3$ & $12,3 \pm 5,0$ \\
Talla (metros) & $1,55 \pm 5,630 \mathrm{E}-02$ & $1,55 \pm 5,779 \mathrm{E}-02$ \\
Hemoglobina (g/dL) & $10,8 \pm 1,2$ & $10,4 \pm 1,5$ \\
Intervalo entre el parto & & \\
y el inicio dela prueba (h) & $21,8 \pm 9,7$ & $16,6 \pm 8,5$ \\
Peso del recién nacido (g) & $3388,7 \pm 259$ & $4216,8 \pm 181,6$ \\
Índice de masa corporal & & \\
inicial (kg/m ${ }^{2}$ ) & $23,8 \pm 4,5$ & $26,3 \pm 3,9$ \\
Índice de masa corporal & & \\
final (kg/m²) & $28,1 \pm 4,2$ & $31,4 \pm 3,9$ \\
Grado de instrucción & & \\
. Superior & $5 \%$ & $12,7 \%$ \\
. Secundaria & $73,2 \%$ & $21,1 \%$ \\
Primaria & $19,7 \%$ & \\
\hline
\end{tabular}

DE: Desviación estándar.

Incluye 1 mes antes del parto y puerperio inmediato. 
Tabla 2. Características obstétricas de la población.

\begin{tabular}{|c|c|c|}
\hline $\begin{array}{l}\text { Característica } \\
\text { obstétrica }\end{array}$ & $\begin{array}{c}\text { Casos } n=71 \\
\text { macrosómicos }\end{array}$ & $\begin{array}{c}\text { Controles } \mathrm{n}=71 \\
\text { no macrosómicos }\end{array}$ \\
\hline $\begin{array}{l}\text { Gestación } \\
\text { (promedio } \pm D E) \\
\text { Primigesta } \\
\text {. Multigesta }\end{array}$ & $\begin{array}{l}2,7 \pm 1,6 \\
29,6 \% \\
70,4 \%\end{array}$ & $\begin{array}{c}2,5 \pm 1,4 \\
26,8 \% \\
73,2 \%\end{array}$ \\
\hline $\begin{array}{l}\text { Paridad } \\
\text { (Promedio } \pm \mathrm{DE}) \\
\text {. Nulipara } \\
\text {. Primipara } \\
\text {. Multipara }\end{array}$ & $\begin{array}{l}1,3 \pm 1,3 \\
33,8 \% \\
26,8 \% \\
39,4 \%\end{array}$ & $\begin{array}{l}1,1 \pm 1,2 \\
36,6 \% \\
36,6 \% \\
26,8 \%\end{array}$ \\
\hline $\begin{array}{l}\text { Edad gestacional } \\
\text { (semanas } \pm D E) \\
\text {. Por FUR } \\
\text { Por ecografía } \\
\text {. Por Capurro }\end{array}$ & $\begin{array}{l}39,9 \pm 1,5 \\
39,9 \pm 1,0 \\
40,1 \pm 0,6\end{array}$ & $\begin{array}{l}39,4 \pm 1,5 \\
39,2 \pm 1,3 \\
39,7 \pm 0,9\end{array}$ \\
\hline $\begin{array}{l}\text { Control prenatal } \\
\text {. Sí } \\
\text {. No }\end{array}$ & $\begin{array}{c}97,2 \% \\
2,8 \%\end{array}$ & $\begin{array}{l}85,9 \% \\
14,1 \%\end{array}$ \\
\hline $\begin{array}{l}\text { Número de CPN } \\
\text { (promedio } \pm \mathrm{DE})\end{array}$ & $7,6 \pm 2,8$ & $6,8 \pm 2,4$ \\
\hline
\end{tabular}

DE: Desviación estándar. FUR: Fecha última de la regla.

$C P N$ : Contro prenatal.

Los resultados del análisis univariado y multivariado se presenta en la Tabla 4. El antecedente de macrosomia fetal es un factor de riesgo para macrosomia fetal en el siguiente embarazo. Un índice de masa corporal inicial mayor o igual de 25 e índice de masa corporal final mayor o igual de 30 constituyen un factor de riesgo, con un OR de 3,1 (IC95\% 1,5-6,1) y OR 6,7 (IC95\% 3,2-14,1). El antecedente de aborto, óbito y preeclampsia no fue estadísticamente

Tabla 3. Validación de hipótesis.

\begin{tabular}{lllll}
\hline & & \multicolumn{2}{c}{ Muestra } & \\
\cline { 3 - 4 } & & Casos & Controles & Total \\
\hline \multirow{2}{*}{ Resultado } & Positivo & 35 & 10 & 45 \\
de la prueba & Negativo & 36 & 61 & 97 \\
& Total & 71 & 71 & 142 \\
\hline Chi-cuadrado $=$ & 20,332 & $p=0,000$ & & \\
OR $=5,931$ & & $95 \% C I=2,626-13,393$ &
\end{tabular}

significativo.

Como la variable dependiente es dicotómica (macrosomia fetal) y las variables independientes son cualitativas y cuantitativas, se utilizó el análisis de regresión logística para poder identificar aquellos factores o características significativas que explicaran la macrosomia. Los resultados se presenta de manera resumida en la Tabla 5.

Ajustando el modelo según los criterios de clasificación teórica de las variables, el modelo de regresión logística es:

Macrosomía fetal (MF)=-4,935+1,710(PTOG)+2,553(ANTC MF)

ANTC MF: antecedente de macrosomia fetal

Después de ajustar la prueba de tolerancia ora a la glucosa con cada uno de los factores asociados con la macrosomia fetal, el OR no se modificó.

\section{DISCUSIÓN}

Relativamente pocos estudios han buscado la relación entre macrosomia fetal y prueba de tolerancia oral a la glucosa en el puerperio para detectar antecedente de diabetes gestacional. Carpenter y col $\left(^{1}\right)$ evaluaron la utilidad de la prueba de tolerancia oral posparto para detectar antecedente de diabetes gestacional. Para ello, validaron una prueba llamada glicemia incremental de 1 y 2 horas, considerándola positiva si se obtenía valores por encima a $110 \mathrm{mg} \%$; con una especificidad de $90,8 \%$ y sensibilidad de $80 \%$ con respecto al antecedente de diabetes gestacional. Concluyeron que los valores de glicemia incremental de 1 y 2 horas a un umbral >ó igual a $110 \mathrm{mg} \%$ asociado a macrosomia diferenciaba a las diabéticas gestacionales de las no diabéticas, y un resultado anormal de esta glicemia incremental justificaría una prueba de tolerancia en futuros embarazos y una vigilancia posterior en la vida de las pacientes. Para el presente estudio, nos amparamos bajo dicho criterio para alcanzar los 
Tabla 4. Factores maternos y obstétricos asociados con macrosomia fetal.

\begin{tabular}{|c|c|c|c|c|c|c|}
\hline \multirow[t]{2}{*}{$\begin{array}{l}\text { Factor materno } \\
\text { u obstétrico }\end{array}$} & \multicolumn{2}{|c|}{$\begin{array}{c}\text { Casos } \mathrm{n}=71 \\
\text { Macrosómicos }\end{array}$} & \multicolumn{2}{|c|}{$\begin{array}{c}\text { Controles } \mathrm{n}=71 \\
\text { No macrosómicos }\end{array}$} & \multirow[b]{2}{*}{ OR } & \multirow[b]{2}{*}{ IC95\% } \\
\hline & $\mathrm{n}$ & $\%$ & $\mathrm{n}$ & $\%$ & & \\
\hline Antecedente de macrosomia fetal & 13 & 18,3 & 1 & 1,4 & 15,7 & $1,9-123,5$ \\
\hline Antecedente de óbito & 2 & 2,8 & 0 & 0,0 & NS & \\
\hline Antecedente de aborto & 19 & 26,8 & 17 & 23,9 & NS & \\
\hline Antecedente de preeclampsia & 2 & 2,8 & 1 & 1,4 & NS & \\
\hline Control prenatal & 69 & 97,2 & 61 & 85,9 & 5,9 & $2,6-13,4$ \\
\hline Aumento de más de $13 \mathrm{~kg}$ & 33 & 46,5 & 20 & 28,2 & 0,5 & $0,2-0,9$ \\
\hline IMC inicial $\geq 25$ & 40 & 56,3 & 21 & 29,6 & 3,1 & $1,5-6,1$ \\
\hline IMC final $\leq 30$ & 47 & 66,2 & 16 & 22,5 & 6,7 & $3,2-14,1$ \\
\hline
\end{tabular}

NS = No significativo.

IMC: índice de masa corporal $\left(\mathrm{kg} / \mathrm{m}^{2}\right)$.

objetivos y demostrar la hipótesis, obteniendo un OR ajustado de 5,9.

Oats y col $\left({ }^{22}\right)$ evaluaron la persistencia de una tolerancia oral a la glucosa alterada en el puerperio, en un estudio de casos y controles realizado en el hospital Mercy Maternity en Melbourne (Australia), en 270 mujeres con diagnóstico previo de diabetes gestacional, en los 7 primeros días posparto. Encontraron que $30 \%$ de las mujeres que presentaron diabetes gestacional tenía una tolerancia oral a la glucosa alterada en el puerperio inmediato. Cabe resaltar que en este trabajo se usó como diagnóstico el criterio estándar de la tolerancia oral a la glucosa y no la tolerancia oral a la glucosa modificada por Carpenter. Además, consideraron para la realización de esta prueba hasta los 7 primeros días posparto. Como es sabido, los niveles altos de glicemia de la diabetes gestacional disminuyen progresivamente una vez concluido el embarazo, descenso que se hace más evidente a las 48 horas. Por lo tanto, a mayores días de puerperio, las posibilidades de tener una tolerancia oral a la glucosa positiva son menores. Oats concluye que si la prueba de tolerancia oral a la glucosa no ha sido realizada prenatalmente,

Tabla 5. Análisis de regresión logística.

\begin{tabular}{llrrrrrrr}
\hline & & & & & & \multicolumn{2}{c}{ IC95\% para Exp(B) } \\
\cline { 3 - 7 } & B & DE & Wald & df & Sig. & Exp(B) & Inferior & Superior \\
\hline a PTOG & 1,710 & 0,470 & 13,253 & 1 & 0,000 & 5,528 & 2,202 & 13,877 \\
IMC1 & $-0,026$ & 0,626 & 0,002 & 1 & 0,967 & 0,974 & 0,286 & 3,321 \\
IMC2 & $-1,515$ & 0,626 & 5,855 & 1 & 0,016 & 0,220 & 0,064 & 0,750 \\
Antcmacr & 2,553 & 1,175 & 4,725 & 1 & 0,030 & 12,850 & 1,286 & 128,45 \\
Deltaw & $-0,073$ & 0,050 & 2,116 & 1 & 0,146 & 0,930 & 0,843 & 1,026 \\
Para & 0,074 & 0,176 & 0,176 & 1 & 0,675 & 1,076 & 0,763 & 1,519 \\
Constante & $-4,935$ & 2,743 & 3,237 & 1 & 0,072 & 0,007 & \\
\hline
\end{tabular}

$a$ : Variable(s) ingresadas en paso 1 DE: Desviación estándar del valor previo df: Grados de libertad $\operatorname{Exp}(B)$ : Operación matemática para calcular el OR PTOG: Prueba de tolerancia oral a la glucosa IMC2: Índice de masa corporal final Deltaw: Peso final menos peso inicial
B: Valor del coeficiente para la variable en prueba $(y=a+B x)$ Wald: Prueba de estabilidad de la regresión logística Sig.: Nivel de significación estadística

OR para esa variable

IMC1: Índice de masa corporal inicial Antcmacr: Antecedente de macrosomia fetal Para: Paridad 
aún es posible realizarla en el puerperio inmediato. Si la posibilidad de diabetes gestacional no se ha hecho evidente con factores relacionados a ella como macrosomia, preeclampsia y muerte fetal. Al igual que la opinión de Oats, estamos de acuerdo en señalar que el tamizaje en el puerperio no es un sustituto del tamizaje gestacional. Bukulmez y col, en el año 1999 ('), valoraron la presencia de un subtítulo de metabolismo anormal en madres con recién nacido macrosómico, utilizando una prueba de tolerancia oral a glucosa en el posparto en un estudio prospectivo de casos y controles. En los resultados de este trabajo, los autores refieren que la mejor predicción de diabetes gestacional podría ser hecha por los valores de glicemia incremental de 1 y 2 horas y los valores de prueba de tolerancia oral en el posparto. Refiere Bukulmez que un umbral $>111 \mathrm{mg} / \mathrm{dL}$ para los valores de glicemia incremental de 1 y 2 horas puede predecir la diabetes gestacional, al igual que la prueba de tolerancia oral a la glucosa en el posparto, y obtiene una sensibilidad de $80 \%$ y una especificidad de $78 \%$, a diferencia de Carpenter que obtuvo un $90 \%$ de especificidad. Concluyen Bukulmez y col que la prueba de tolerancia oral a la glucosa en el posparto puede identificar a un grupo de mujeres con recién nacidos macrosómicos que presentan alteraciones de un estado prediabético y es necesario tomar medidas preventivas para el desarrollo evidente de la diabetes en ellas.

En un estudio local el año 1995, Pacora $\left({ }^{23}\right)$ concluye que el valor promedio de la prueba de tolerancia oral a la glucosa con $50 \mathrm{~g}>100 \mathrm{mg} / \mathrm{dL}$ está asociado con un significativo mayor riesgo materno y perinatal, entre ellos: cesárea, distocia de hombros, desproporción cefalopélvica y macrosomia, como un nuevo criterio para la evaluación de la intolerancia gestacional a la glucosa en la mujer peruana.

Coincidimos con Pacora, Roman y Ardiles al señalar que el antecedente de macrosomia fetal constituye un predictor de riesgo en la futura madre. Encontramos un OR de 15,7 (IC95\% 1,9-123,6) $\left({ }^{13-15,17,19}\right)$. Sin embargo, Añaños $\left({ }^{4}\right)$, en un estudio prospectivo descriptivo y transversal, con criterios de inclusión discutibles, no encuentra recién nacido macrosómico en las gestantes con diabetes gestacional. Navarrete ( $)$ ), en un estudio sobre prevalencia de diabetes gestacional, halla recién nacido macrosómico en $50 \%$ de las gestantes diagnosticadas de diabetes gestacional.

No encontramos asociación estadística entre macrosomia con sobrepeso (>13 kg), antecedente de aborto, preeclampsia, antecedente de óbito (Tabla 4), contrariamente a lo señalado por otros autores $\left({ }^{7}\right)$; pero, sí con el control prenatal, con un OR 5,7 (IC95\% 1,2-26,8); evidentemente, esta "asociación" está por el contexto del antecedente de macrosomia, lo cual induce a un mayor control prenatal. Esto se pudo corroborar al realizar el análisis de regresión logística en el que se consideró a todas estas variables como confusoras (Tabla 5), obteniéndose una expresión macrosomia $=-4,935$ $+1,710$ PTOG + 2,553 (antecedente de macrosomia fetal).

Adicionalmente, queremos señalar que no se encontró asociación, dado el tamaño de la muestra, entre antecedente de aborto, óbito, preeclampsia, obesidad, ganancia ponderal excesiva; pero, sí con el antecedente de macrosomia fetal -aunque el tamaño muestral no fue suficiente-e índice de masa corporal, aspectos que parecen tener una importancia gravitante en el desarrollo de la enfermedad.

Se debería realizar mayores estudios diagnósticos en aquellas gestantes con antecedente de macrosomia fetal para así evitar la diabetes gestacional y sus consecuencias.

Determinar fehacientemente el grado de asociación entre el antecedente de macrosomía fetal y diabetes gestacional representa una limitación del trabajo, aunque no fue el objetivo de este trabajo; aún así resultaría interesante saber con mayor exactitud en cuanto influye el antecedente de 1,2 ó más macrosómicos para producir diabetes gestacional en el siguiente embarazo.

En conclusión, existe una relación entre la prueba de tolerancia oral a la glucosa modificado 
por Carpenter en las puérperas con recién nacidos macrosómicos en el IEMP, siendo ésta un método para el diagnóstico retrospectivo de diabetes gestacional en aquellas gestantes con un adecuado control prenatal pero que no fueron sometidas a la prueba.

\section{AGRADECIMIENTOS}

Los autores desean agradecer a la Dra. Ylia Espinoza Vivas, Directora de la Dirección Ejecutiva de Investigación y Desarrollo de Tecnologías IEMP; Dr. Ricardo Alvarez, Jefe del Laboratorio de Emergencia del IEMP, Dr. Andrés Lucen Zárate, Jefe del departamento de Patología y Laboratorio IEMP que hicieron posible la realización del presente trabajo, a través de la ayuda técnica.

\section{BIBLIOGRAFÍA}

1. Bukulmez O, Durukan T. Postpartum oral glucose tests in mothers of macrosomic infants: inadequacy of current antenatal test criteria in detecting prediabetic state. Europ J Obstet Gynecol Reprod Biol 1999; 86: 29-34.

1. Patiño G. Prueba de tolerancia a la glucosa oral en 100 gestantes atendidas en el Hospital de Apoyo de YanahuaraIPSS de Arequipa. (Tesis de Bachiller en Tecnología Médica), Lima-Perú, Universidad Peruana Cayetano Heredia, 1989.

2. Navarrete V. Prevalencia de diabetes gestacional en el Hospital Nacional Guillermo Almenara Irigoyen. (Tesis Bachiller Tecnología Médica). Lima-Perú. Universidad Peruana Cayetano Heredia, 1993.

3. Añaños M. Prevalencia de diabetes mellitus gestacional en el Hospital Nacional Cayetano Heredia.2002. (Tesis de Bachiller en Medicina). Lima-Perú. Universidad Peruana Cayetano Heredia. 2002.

4. Oficina de Estadística e informática del Instituto Especializado Materno Perinatal. Informe anual del año 2,002. Lima- Perú.

6. Larson G, Spjuth J, Ramstam J, Vikbladh I, axtrup O, Astedt B. Prognostic significance of birth of large infant for subsequent development of maternal non-insulin-dependent diabetes mellitus: a prospective study over 20-27 years. Diabetes Care 1986; 9: 359-64.

7. Arias F. Diabetes y Embarazo. En: Guía práctica par el embarazo y el parto de alto riesgo. Segunda Edición. Madrid: Mosby/Doyma Libros. 1994: 284-303.

8. Summary and Recommendations of the Fourth International workshop-Conference on Gestational Diabetes Mellitus. Diabetes Care 1998; 21 (suppl. 2): 3121- 67.
9. Boyd ME, Usher RH, Mc Lean FH. Fetal macrosomia: Prediction risks proposed management. Obstet Gynecol 1983; 61: 715-21.

10. Pederson J. The Pregnant Diabetic and her Newborn: Problems and Management. $2^{\text {nd }}$ edition. Baltimore, MD: Williams y Wilkins, 1977: 211-20.

11. Michael D, Debra C, Oded L. El feto macrosómico. Clín Obstét Ginecol 1999; 4.

12. American Diabetes Association. Gestational diabetes mellitus. Diabetes Care. 2003; 26 (1): 103-5.

13. Román V. Macrosomía: características maternas y complicaciones neonatales. (Tesis de Bachiller en Medicina). Lima-Perú. Universidad Peruana Cayetano Heredia. 1990.

14. Uvena-Celebrezze J, Catalano P. El hijo de la diabética gestacional. Clín Obstét Ginecol 2000; 1: 117-27.

15. Tamura R, Dooley S . Ultrasonografía en el tratamiento de diabéticas embarazadas. Clín Obstét Ginecol 1991; 3: 511-40.

16. Kjos SL, Peters RK, Xiang A, Henry OA, Montoro M, Buchanan TA. Predicting future diabetes in Latino women with gestational diabetes. Utility of early post partum glucose tolerance testing. Diabetes 1995; 44: 586.

17. Pacora P. Macrosomía fetal: definición, predicción, riesgos y prevención. Ginecol Obstet (Perú) 1994; 39: 42-50.

18. Pacora P. Diabetes y embarazo: Vigilancia epidemiológica. Ginecol Obstet (Perú) 1996;42:10-20.

19. Ardiles T. Factores asociados a macrosomía fetal. (Tesis de Bachiller en Medicina). Lima-Perú. Universidad Peruana Cayetano Heredia. 2002.

20. Ministerio de Salud. Instituto Materno Perinatal. Manual de normas y procedimientos en la atención obstétrica.1995.

21. Carpenter M, Coustan D, Gruppuso P, Malone M, Rotondo L. Postpartum testing for antecedent gestacional diabetes. Am Jl Obstet Gynecol 1988; 159: 1128-31.

22. Oats J, Beischer A. The persistence of abnormal glucose tolerance after delivery. Part 1. Obstet Gynecol 1990; 75.

23. Pacora P. Un nuevo criterio para la evaluación de la intolerancia gestacional a la glucosa en la mujer peruana. Diagnóstico 1995; 34: 15-21. 